\title{
Funcionalidade e risco de quedas de idosos participantes de um grupo de convivência de Flores da Cunha, RS
}

\section{Functionality and risk of falls of elderly participants of a companionship group of Flores da Cunha, RS}

\author{
Gabriela Oliboni Fiorio', Gisele Oltramari Meneghini' \\ ' Centro Universitário da Serra Gaúcha (FSG), Caxias do Sul, RS, Brasil.
}

\section{ARTICLE INFO}

\section{Article history}

Received: 10/07/2018

Accepted: 16/11/2018

\section{Correspondent Author}

Gabriela Oliboni Fiorio

Av. Rubem Bento Alves, 4548/301

95032-318 Caxias do Sul, RS, Brasil

(D) orcid.org/0000-0001-6532-0310

<fiorio.gabriela@gmail.com>

(C) 2018 All rights reserved

\section{Editors}

Alfredo Cataldo Neto

Paula Engroff

\begin{abstract}
RESUMO
INTRODUÇÃO: $\bigcirc$ processo natural de envelhecimento gera diversas consequências sejam físicas, psicológicas e sociais, podendo afetar a funcionalidade e aumentando a incidência de quedas. Estas apresentam diversos impactos na vida de um idoso, como deterioração funcional, hospitalização e institucionalização. OBJETIVO: Avaliar a funcionalidade e o risco de quedas de idosos participantes de um grupo de convivência. MÉTODOS: Tratou-se de um estudo transversal, onde os participantes eram idosos integrantes de um grupo de convivência de Flores da Cunha, RS. A coleta de dados foi efetuada por meio da aplicação de quatro instrumentos de avaliação, sendo eles uma ficha inicial de avaliação, Escala de Lawton, Teste de Alcance Funcional Anterior (TAF) e Timed Up and Go Test (TUGG). RESULTADOS: Mesmo por se tratar de idosos ativos, a maioria dos entrevistados afirmou não realizar atividade física. 86,8\% da amostra estudada representou ser independente para realizar as atividades instrumentais de vida diária, conforme pontuação na Escala de Lawton. Já nos Testes Alcance Funcional (TAF) e Timed Up and Go Test (TUG), 99,1\% dos idosos participantes do estudo deslocaram-se dentro do limite normal de estabilidade anterior e apresentaram baixo risco para quedas. CONCLUSÃO: Quando correlacionadas entre si, a Escala de Lawton, o TAF, - TUG e a idade, mostra-se que o avanço da idade, a capacidade funcional, o equilíbrio, a mobilidade e o risco de quedas estão diretamente associados uns com os outros. Portanto, conclui-se que a maioria dos idosos participantes de grupos de convivência apresentam bom desempenho nos testes funcionais.
\end{abstract}

DESCRITORES: idoso; incapacidade e saúde; acidentes por quedas.

\begin{abstract}
INTRODUCTION: The natural aging process generates several physical, psychological and social consequences, which can affect the functionality and increase the incidence of falls. These have several impacts on the life of an elderly person, such as functional deterioration, hospitalization and institutionalization. OBJECTIVE: To evaluate the functionality and risk of falls of elderly participants in a companionship group. METHODS: This was a cross-sectional study, in which the participants were elderly belonging to a companionship group from Flores da Cunha, RS. Data collection was done through the application of four evaluation instruments, including an initial evaluation card, Lawton Scale, Previous Functional Reach Test (TAF) and Timed Up and Go Test (TUG). RESULTS: Even though the elderly were active, most interviewees said they did not perform physical activity. $86.8 \%$ of the studied sample was independent to perform instrumental activities of daily living, according to the Lawton Scale score. On the Functional Reach Test (TAF) and Timed Up and Go Test (TUG), 99.1\% of the study participants moved within the normal limit of previous stability and presented a low risk for falls. CONCLUSION: When correlated with each other, the Lawton Scale, TAF, TUG and age, it is shown that the advancement of age, functional capacity, balance, mobility and risk of falls are directly associated with others. Therefore, it is concluded that the majority of the elderly participants of companionship group present good performance in the functional tests.

KEYWORDS: elderly; disability and health; accidents by falls.
\end{abstract}




\section{INTRODUÇÃO}

O envelhecimento populacional é um fenômeno que vem influenciando bastante a estrutura demográfica mundial. Presente tanto nos países desenvolvidos quanto naqueles em desenvolvimento, é um processo relacionado com a redução da taxa de fecundidade e o aumento da expectativa de vida, o que gera, consequentemente, diminuição da mortalidade da população idosa. $\mathrm{O}$ número de pessoas com 60 anos ou mais está crescendo de forma acelerada mais do que qualquer outra faixa etária. Segundo Camarano ${ }^{1}$, estima-se que em 2020 haja em torno de 30,9 milhões de pessoas com idade superior a 60 anos no Brasil, tornando-se o sexto país do mundo em número de idosos.

Para a Organização Pan-Americana da Saúde $\mathrm{OPAS}^{2}$, melhorar a qualidade de vida à medida que as pessoas entram para o grupo da terceira idade, incentivar sua participação no meio social, promover segurança e oferecer oportunidades de saúde são objetivos fundamentais para um envelhecimento ativo. Os grupos de convivência têm sido uma alternativa bastante estimulada em todo o Brasil para suprir esses quesitos ${ }^{3}$.

Segundo Andrade et al..$^{4}$, os grupos de convivência para idosos contribuem para a promoção de um envelhecimento ativo e a preservação das capacidades e do potencial do indivíduo nessa fase. É também um importante espaço para desencadear na pessoa idosa e na comunidade uma mudança comportamental, posto que essa relação ainda é vista, por muitos, como uma situação de preconceito. Sendo assim, o fortalecimento do papel social do indivíduo idoso é um dos principais objetivos visados pelos atuais projetos de convivência ${ }^{5}$.

A incapacidade funcional, um dos comprometimentos advindos com o avanço cronológico da idade, é caracterizada como qualquer restrição que interfira no desempenho de uma atividade dentro da extensão considerada normal para a vida humana. Apontada também por ser uma influente condição de mortalidade na população idosa, deve-se incluí-la como um método avaliativo por profissionais que lidam com essa faixa etária, visando a alcançar medidas preventivas e até mesmo intervenções terapêuticas que reduzam os mecanismos influentes no processo de declínio das habilidades físicas e mentais cotidianas ${ }^{6}$.

Barboza et al. ${ }^{7}$ afirmam que a mobilidade física alterada desencadeia diminuição e até mesmo perda da capacidade funcional, desde atividades simples até as mais complexas. Para Gonçalves ${ }^{8}$, as alterações físicas que acontecem no organismo humano na fase da velhice fazem com que ao longo dos anos os tecidos percam a flexibilidade e a capacidade de recuperação, passando a produzir baixa imunidade frente às doenças, tornando os órgãos e sistemas corporais mais lentos e, consequentemente, afetando suas funções. $\mathrm{O}$ processo natural de envelhecimento gera uma diminuição da massa e força muscular, fato esse também conhecido como sarcopenia, o que, por conseguinte, afeta a funcionalidade, a qualidade de vida, o aumento da dependência e da incidência de quedas ${ }^{9}$.

As quedas apresentam diversos impactos na vida de um idoso, como, por exemplo, morbidade importante, mortalidade, deterioração funcional, hospitalização, institucionalização e consumo de serviços sociais e de saúde ${ }^{10}$. Em suma, avaliar o risco de quedas e orientar o idoso e seus familiares sobre os impactos e suas consequências pode fazer a diferença para evitar esse tipo de situação, retardando assim as limitações funcionais, a diminuição da autonomia e independência, além de quadros depressivos e baixa qualidade de vida nos indivíduos da terceira idade ${ }^{11}$.

Sabendo-se que as quedas representam um evento que acarreta inúmeras consequências na vida de um idoso, fez-se necessário avaliar os fatores preditores como nível de funcionalidade e risco de quedas, além da prática ou não de atividade física e faixa etária de uma população específica de idosos do município de Flores da Cunha, RS.

\section{MÉTODO}

Esta pesquisa tratou-se de um estudo transversal. A amostra, recrutada por conveniência, foi constituída por indivíduos de ambos os sexos, com faixa etária entre 60 e 91 anos, participantes de um grupo de convivência de Flores da Cunha, RS, denominado "Projeto Conviver". Este projeto é coordenado pela Secretaria de Desenvolvimento Social, através do CRAS - Centro de Referência em Assistência Social. Os encontros são semanais e acontecem nas tardes de quarta-feira, no Salão Paroquial de Flores da Cunha, local onde foram realizadas as coletas dos dados. $\mathrm{O}$ programa visa à reinserção dos idosos no convívio social por meio de atividades de confraternização, a fim de oferecer à terceira idade uma melhor qualidade de vida.

A coleta de dados foi efetuada por meio da aplicação de quatro instrumentos de avaliação, sendo eles uma ficha inicial de avaliação, contendo os dados de identificação e características sociodemográficas e clínicas, a Escala de Lawton, para avaliar a funcionalidade, e os Testes de Alcance Funcional Anterior (TAF) e Timed Up and Go Test (TUG), ambos para avaliar o risco de quedas. 
Inicialmente, foi realizada a coleta de dados de identificação de cada idoso participante, por meio de uma Ficha de Avaliação elaborada pelos pesquisadores, contendo na mesma dados de identificação pessoal e características sociodemográficas e clínicas, como sexo, idade, estado civil, escolaridade, prática de atividade física, patologias pregressas e/ou recentes, condições do ambiente domiciliar, histórico de quedas e suas consequências após o evento, além de questionamentos relacionados a possíveis fatores que poderiam interferir na realização das suas atividades de vida diária (AVD's).

Em seguida, foi avaliado o nível de funcionalidade dos participantes por meio da Escala de Lawton, na qual se avalia o desempenho do idoso em relação às atividades instrumentais de vida diária, a fim de verificar a sua independência funcional. Escala essa desenvolvida e validada em 1969 por Lawton e Brody ${ }^{12}$. Os indivíduos participantes foram classificados como independentes ou dependentes no desempenho de nove funções, sendo elas: usar o telefone celular, ir a locais distantes usando algum transporte e sem necessidade de planejamentos especiais, fazer compras, preparar as refeições, arrumar a casa, realizar trabalhos manuais domésticos, lavar e passar a roupa, manejar seus remédios e controlar as finanças. A pontuação varia de 9 a 27 pontos, considerando que, para cada questão, a primeira resposta significa independência ( 3 pontos), a segunda, dependência parcial ou capacidade com ajuda ( 2 pontos) e a terceira, dependência (1 ponto). As questões 4 a 7 podiam ter variações conforme o sexo e podiam ser adaptadas para atividades como subir escadas ou cuidar do jardim ${ }^{13}$.

$\mathrm{Na}$ sequência, para identificar o risco de quedas, foi realizado o Teste de Alcance Funcional Anterior (TAF), com o intuito de determinar o quanto o idoso era capaz de se deslocar dentro do limite de estabilidade anterior. Conforme Karuka et al. ${ }^{14}$, a fita métrica é presa em uma parede, paralela ao chão e posicionada na altura do acrômio do participante. Descalço, o indivíduo foi posicionado com os pés confortáveis e paralelos entre si, perpendicularmente em relação à parede e próximos ao início da fita métrica. Mantendo os punhos em posição neutra, cotovelos estendidos e ombros com flexão de $90^{\circ}$, o voluntário foi instruído a realizar uma inclinação para frente sem tocar na fita e, logo após, verificou-se o deslocamento realizado sobre a mesma. Tem-se o resultado do teste após três tentativas, por meio da média da diferença entre a medida na posição inicial e a final registrada na régua. Utilizou-se a referência de Gomes ${ }^{15}$, que afirma que deslocamentos menores que $15 \mathrm{~cm}$ indicam fragilidade do paciente e risco de quedas.
O último teste realizado foi o Timed Up and Go Test (TUG), instrumento utilizado para complementar a avaliação do risco de quedas. Segundo Podsiadlo e Richardson ${ }^{16}$, o TUG tem como objetivo avaliar a mobilidade e o equilíbrio funcional. $O$ teste quantifica em segundos a mobilidade funcional por meio do tempo que o indivíduo realiza a tarefa de levantar de uma cadeira sem ajuda dos braços, caminhar 3 metros, virar, voltar rumo à cadeira e sentar novamente. Karuka et al. ${ }^{14}$, afirmam que, no TUG, o idoso parte da posição inicial com as costas apoiadas na cadeira. A cronometragem foi iniciada após o sinal de partida e parada somente quando o idoso se colocou novamente na posição inicial, sentado com as costas apoiadas na cadeira. Considerou-se que a realização do teste em até 10 segundos foi o tempo considerado normal para adultos saudáveis, independentes e sem risco de quedas. Valores entre 11-20 segundos é o esperado para idosos com deficiência ou frágeis, com independência parcial e com baixo risco de quedas. Já acima de 20 segundos sugere que o idoso apresenta déficit importante da mobilidade física e risco de quedas. Determina-se um desempenho de até 12 segundos como tempo normal de realização do teste para idosos comunitários ${ }^{17}$.

Após a aprovação do Comitê de Ética em Pesquisa da FSG, sob o Parecer de número 2.080.084, aceito no dia 24 de Maio de 2017, todos os participantes receberam uma breve explicação sobre a pesquisa realizada e, após a concordância do indivíduo e a assinatura do Termo de Consentimento Livre e Esclarecido, foi iniciada a coleta de dados.

Foram excluídos da pesquisa participantes que não adquiriam o ortostatismo e não possuíam capacidades físicas para realizar os testes propostos, impedindo assim sua aplicabilidade, e também, aqueles que se negarem a assinar o Termo de Consentimento Livre e Esclarecido. A coleta de dados teve um período de duração de, aproximadamente, três meses, sendo iniciada no dia 31 de maio de 2017 a 30 de agosto do mesmo ano.

Para análise estatística, foram utilizadas estatísticas descritivas para caracterização da amostra, que serão descritas como média, desvio padrão e valores mínimos e máximos. Foi usado o teste qui quadrado para comparar as variáveis selecionadas entre os grupos de idade da amostra avaliada. De acordo com a distribuição paramétrica dos dados, utilizou-se o coeficiente de correlação de Pearson para verificar as associações entre idade, TUG, Lawton e TAF. De acordo com Portney e Watkins ${ }^{25}$, as correlações foram classificadas como pouca ou nenhuma $(0,00<\mathrm{r}<0,25)$, razoável $(0,25<\mathrm{r}<0,50)$, moderada a boa $(0,50<\mathrm{r}<0,75)$ e boa a excelente $(r>0,75)$. Todas as análises foram realizadas utilizando o programa estatístico SPSS para Windows, 
versão 17.0, e o nível de significância estabelecido foi $\alpha=0,05$.

\section{RESULTADOS}

Fizeram parte da amostra 114 idosos. A Tabela 1 demonstra a estratificação dos indivíduos de acordo com a faixa etária, tendo uma média de idade de 72,5 $\pm 6,58$ (60-91) anos. A maioria era do sexo feminino. Mais da metade dos entrevistados relataram serem viúvos e não morarem sozinhos.

Tabela 1. Estratificação dos indivíduos de acordo com a faixa etária e atividade física realizada ou não pela população estudada.

\begin{tabular}{lcc}
\hline & Frequência & \% \\
Idade (anos) & & \\
60 a 69 & 41 & 36,0 \\
70 a 79 & 54 & 47,4 \\
80 a 89 & 17 & 14,8 \\
$\geq 90$ & 2 & 1,8 \\
Atividade física & & \\
$\quad$ Sim (Caminhadas, Ginástica) & 48 & 42, \\
Não & 66 & 57,9 \\
\hline
\end{tabular}

Conforme o perfil de escolaridade da amostra estudada, a maior parte dos idosos pertencentes ao grupo de convivência de Flores da Cunha, RS tivera completado até a 4⿳亠丷a série, conhecido também como Primário Completo.

Quando questionados sobre a prática de atividade física, 57,9\% da população entrevistada relataram não realizar atividade física. Já aqueles que afirmaram serem bastante ativos, alegaram a prática de caminhadas diárias e ginástica, com frequência semanal de, aproximadamente, 2 a 3 vezes por semana. Ambos os grupos de idosos, levando em consideração a média obtida, tiveram resultados satisfatórios em relação às variáveis risco de quedas e funcionalidade.

Todos os indivíduos relataram possuir iluminação adequada. 57,9\% relataram que o banheiro não era adaptado. $40,4 \%$ relataram que os tapetes eram antiderrapantes, $23,7 \%$ que eram escorregadios e $36 \%$ relataram não possuir tapetes. A maioria (76,3\%) relatou haver poucos degraus em casa. Todos os indivíduos relataram que a locomoção é fácil. 80,7\% relataram que não apresentaram quedas no último ano. 15,8\% tiveram queda nos últimos 3 meses e 3,5\% relataram mais de uma queda nos últimos 3 meses.

Com relação à presença de dor, 46,5\% dos indivíduos relataram sentir dor, principalmente nos membros inferiores, sendo a artrose a principal patologia citada. Ademais, somente 3,5\% da população referiu ter sofrido queda recente. Ao observar a relação da Escala de Lawton, 86,8\% dos idosos foram classificados como Independentes, ou seja, mesmo com presença de dor, alegaram que continuam realizando suas atividades diárias como fazer compras, arrumar a casa, preparar as próprias refeições, entre outras. Já na mensuração do Teste do Alcance Funcional Anterior, 99,1\% da população apresentou classificação Normal, ou seja, deslocaram-se anteriormente mais que $15 \mathrm{~cm}$. Por conseguinte, no Timed Up and Go Test, 99,1\% dos idosos apresentaram baixo risco de quedas.

O teste qui quadrado revelou existir diferenças estatisticamente significativas entre as faixas etárias em relação à Escala de Lawton $(p=0,009)$, conforme demonstra a Tabela 2. Já em relação ao Teste do Alcance Funcional Anterior, o teste qui quadrado revelou não existir diferenças estatisticamente significativas entre grupos estudados, conforme demonstra a Tabela 3. Da mesma forma, em relação ao Timed Up and Go Test, o teste qui quadrado revelou não existir diferenças estatisticamente significativas entre grupos estudados, conforme demonstra a Tabela 4.

Tabela 2. Comparação da Escala de Lawton nas diferentes faixas etárias dos indivíduos incluídos no presente estudo.

\begin{tabular}{|c|c|c|c|c|}
\hline \multirow{2}{*}{$\begin{array}{l}\text { Idade } \\
\text { (anos) }\end{array}$} & \multirow[b]{2}{*}{ Classificação } & \multicolumn{2}{|c|}{ Escala de Lawton } & \multirow[b]{2}{*}{$p$} \\
\hline & & Independente & $\begin{array}{c}\text { Moderamente } \\
\text { Dependente }\end{array}$ & \\
\hline \multirow[t]{2}{*}{60 a 69} & Observado & 37 & 4 & \multirow{8}{*}{0,009} \\
\hline & Esperado & 35,6 & 5,4 & \\
\hline \multirow[t]{2}{*}{70 a 79} & Observado & 50 & 4 & \\
\hline & Esperado & 46,9 & 7,1 & \\
\hline \multirow[t]{2}{*}{80 a 89} & Observado & 11 & 6 & \\
\hline & Esperado & 14,8 & 2,2 & \\
\hline \multirow[t]{2}{*}{$\geq 90$} & Observado & 1 & 1 & \\
\hline & Esperado & 1,7 & ,3 & \\
\hline
\end{tabular}

Tabela 3. Comparação do Teste do Alcance Funcional Anterior nas diferentes faixas etárias dos indivíduos incluídos no presente estudo.

\begin{tabular}{ccccc}
\hline $\begin{array}{c}\text { Idade } \\
\text { (anos) }\end{array}$ & Classificação & Normal & RAF & $\boldsymbol{p}$ \\
\hline 60 a 69 & Observado & 41 & 0 & \\
& Esperado & 40,6 &, 4 & \\
70 a 79 & Observado & 54 & 0 & \\
& Esperado & 53,5 &, 5 & 0,124 \\
80 a 89 & Observado & 16 & 1 & \\
& Esperado & 16,9 &, 1 & \\
$\geq 90$ & Observado & 2 & 0 & \\
& Esperado & 2,0 &, 0 & \\
\hline
\end{tabular}

* Teste do Qui-quadrado para verificar as associações entre idade e TAF. 
Tabela 4. Comparação do Timed Up and Go Test nas diferentes faixas etárias dos indivíduos incluídos no presente estudo.

\begin{tabular}{|c|c|c|c|c|}
\hline \multirow{2}{*}{$\begin{array}{l}\text { Idade } \\
\text { (anos) }\end{array}$} & \multirow[b]{2}{*}{ Classificação } & \multicolumn{2}{|c|}{ Timed Up and Go Test - TUG } & \multirow[b]{2}{*}{$p$} \\
\hline & & $\begin{array}{l}\text { BAIXO risco } \\
\text { para quedas }\end{array}$ & $\begin{array}{c}\text { MÉDIO risco } \\
\text { para quedas }\end{array}$ & \\
\hline \multirow[t]{2}{*}{60 a 69} & Observado & 40 & 1 & \multirow{8}{*}{0,616} \\
\hline & Esperado & 40,6 &, 4 & \\
\hline \multirow[t]{2}{*}{70 a 79} & Observado & 54 & 0 & \\
\hline & Esperado & 53,5 &, 5 & \\
\hline \multirow[t]{2}{*}{80 a 89} & Observado & 17 & 0 & \\
\hline & Esperado & 16,9 &, 1 & \\
\hline \multirow[t]{2}{*}{$\geq 90$} & Observado & 2 & 0 & \\
\hline & Esperado & 2,0 &, 0 & \\
\hline
\end{tabular}

* Teste do Qui-quadrado para verificar as associações entre idade e TUG.

Conforme demonstra a Tabela 5, observou-se correlação estatisticamente significativa, de magnitude pouca a razoável, entre Escala de Lawton, Teste do Alcance Funcional, Timed Up and Go Test e idade da amostra estudada.

Tabela 5. Correlação entre a Escala de Lawton, Teste do Alcance Funcional, Timed Up and Go Test e idade.

\begin{tabular}{cccccc}
\hline \multirow{2}{*}{ Lawton } & & Lawton & TAF & TUG & Idade \\
& $p$ & - &, $226^{*}$ &,$- 339^{* *}$ &,$- 368^{* *}$ \\
\multirow{2}{*}{ TAF } & $p$ & &, 016 &, 000 &, 000 \\
& $\mathrm{r}$ &, $226 *$ & - &,$- 242^{* *}$ &,$- 242^{* *}$ \\
\multirow{2}{*}{ TUG } & $p$ &, 016 & &, 009 &, 009 \\
& $\mathrm{r}$ &,$- 339 * *$ &,$- 242 * *$ & - &, $361 * *$ \\
Idade & $p$ &, 000 &, 009 & &, 000 \\
& $\mathrm{r}$ &,$- 368 * *$ &,$- 242 * *$ &, $361 * *$ & - \\
& $p$ &, 000 &, 009 &, 000 & \\
\hline
\end{tabular}

* Coeficiente de correlação de Pearson para verificar as associações entre idade, TAF, TUG e Lawton.

\section{DISCUSSÃO}

Segundo a Organização Mundial de Saúde (OMS) ${ }^{18}$, até 2025 , o Brasil será o sexto país do mundo em número de idosos. Projeções demográficas apontam que indivíduos acima de 60 anos, em 2047, ultrapassarão, pela primeira vez, o número de crianças no mundo. Levando em consideração esse dado, a média de idade dos 114 idosos participantes da pesquisa foi de $72,5 \pm$, sendo a menor faixa etária de 60 anos e a maior de 91 anos.

Quando questionados sobre a prática ou não de atividade física além do Projeto Conviver, 57,9\% da amostra relataram não realizar. Miranda et al ${ }^{19}$ relatam em seus estudos sobre fatores associados à boa qualidade de vida de idosos de um Centro de Referência, em Belo Horizonte, MG, que o avanço da idade e a atividade física, correlacionada com a qualidade de vida, estão intimamente ligadas no quesito envelhecimento ativo e saudável. A pesquisa evidenciou que idosos que se exercitavam com maior frequência obtiveram médias mais altas nos domínios psicológicos e físicos, apresentando-se mais satisfeitos com sua própria qualidade de vida. A prática de atividade física auxilia no combate do sedentarismo, contribuindo assim para a manutenção da aptidão física do indivíduo idoso. À vista disso, reduz-se a perda de massa muscular, além de melhorar a mobilidade funcional, o equilíbrio, a agilidade durante deambulação e a coordenação ${ }^{20}$.

Em relação aos fatores limitantes para a realização de AVD's, 46,5\% dos idosos afirmaram a presença de dor como principal causa, porém relataram que, mesmo com dor, continuam realizando duas tarefas diárias conforme conseguem. Celich e Galon ${ }^{21}$ afirmam que muitos idosos sentem dor e acabam convivendo diariamente com ela, com o intuito de manter uma vida normal. Isso é justificado pelos autores, os quais discutem o fato de que, muito provavelmente, os idosos já se acostumaram com a presença da dor e a própria não valorização da mesma, visto que há, ainda, a ideia de que a convivência com a dor e a incapacidade funcional é algo extremamente normal, principalmente nessa faixa etária.

De acordo com os resultados, $86,8 \%$ da amostra estudada representaram ser independentes para a realização das AIVD conforme pontuação na Escala de Lawton. Gomercindo e Garcez ${ }^{22}$, em um estudo comparativo sobre a avaliação da capacidade funcional de idosos que praticam regularmente algum tipo de atividade física e idosos sedentários afirmaram que existe significativa diferença na manutenção da capacidade funcional em executar as ABVD's entre idosos ativos e sedentários. Em contrapartida, a realização das AIVD's depende de outros fatores, os quais não foram investigados nesta pesquisa, porém destaca-se que não se relacionam apenas com o fato de o indivíduo idoso ser sedentário ou não. Já Barbosa ${ }^{23}$ explica essa afirmação devido às AIVD's exigirem, além de maior integridade física, também a integridade cognitiva quando comparadas às ABVD's.

No Teste de Alcance Funcional (TAF), apenas 1 dos 114 indivíduos teve média de deslocamento inferior a $15 \mathrm{~cm}$, enquanto que $99,1 \%$ dos idosos participantes do estudo deslocaram-se dentro do limite normal de estabilidade anterior. Segundo Campos et al. ${ }^{24}$, os menores escores do TAF geralmente estão relacionados a fatores como quedas recentes e fraturas, 
muito provavelmente não apenas devido a déficits de equilíbrio, mas também devido a alterações na marcha e diminuição de força muscular e da mobilidade, além de idosos que residem em instituições de longa permanência, e ao sedentarismo. Esses aspectos opõem-se aos das pessoas idosas que frequentam grupos de convivência, visto que as mesmas praticam diversas atividades nesses Projetos, tratando-se de indivíduos com estilo de vida mais ativo, podendo justificar, portanto, os excelentes resultados na avaliação através do TAF.

Quando se fala na variável índice de quedas, Maciel e Araújo ${ }^{25}$, relatam que elas são responsáveis por até $70 \%$ das mortes acidentais em pessoas com mais de 75 anos e estão evidenciadas como as consequências mais perigosas quando há desequilíbrios, déficits posturais e dificuldade de locomoção, muitas vezes deixando os idosos acamados por dias ou até mesmo meses. No presente estudo, ao serem questionados sobre quedas nos últimos meses, $80,7 \%$ dos entrevistados relataram que não apresentaram quedas no último ano, 15,8\% tiveram quedas nos últimos 3 meses e apenas 3,5\% dos participantes do estudo relataram mais de uma queda nos últimos 3 meses. Resultado esse que surpreende quando se trata da população da terceira idade, visto que, conforme citado anteriormente, o risco de quedas aumenta com o avanço da idade.

Durante a realização do Timed Up and Go Test, 99,1\% dos participantes da pesquisa apresentaram baixo risco para quedas, enquanto que apenas 1 indivíduo apresentou médio risco para quedas. Lustosa et al. ${ }^{26} \mathrm{em}$ um estudo sobre a relação da capacidade e o desempenho funcional em idosos frequentadores de grupos de convivência em Belo Horizonte, MG, observaram, na realização do TUG, que a maioria dos idosos avaliados executou o teste abaixo do ponto de corte, o que significa índices mínimos de alteração da mobilidade e dependência funcional. No entanto, vale ressaltar que mesmo esses indivíduos apresentando baixo risco de quedas, a possibilidade de cair ainda existe $^{27}$.

Quando se correlacionou a escala de Lawton com a idade dos indivíduos, observou-se diferença estatisticamente significativa e correlação de magnitude pouca a razoável, o que significa que o indivíduo que tem uma idade menor é mais independente nas suas AVD's. Del Duca et al. ${ }^{6}$ afirmam em seus estudos que, tanto as ABVD's quanto as AIVD's, associam-se com o avanço da idade, aliado ao próprio processo de envelhecimento, o que vem influenciando diretamente na ocorrência de maiores níveis de incapacidade funcional. Lustosa et al. ${ }^{26}$ citam na sua pesquisa, com interpretação de resultados similares a essa, que era de se esperar que idosos frequentadores de grupos de convivência apresentassem bom desempenho nos testes funcionais, visto que se trata de indivíduos mais ativos e com maior independência. Salienta-se também que o nível de independência funcional é diretamente afetado através do estilo de vida adotado pelo idoso ${ }^{27}$.

Ao correlacionar a escala de Lawton, o TUG e a idade, observou-se que aquele indivíduo que tem uma independência maior nas suas AVD's apresenta uma mobilidade funcional melhor e menor risco de quedas. Segundo Souza et al. ${ }^{28}$, conforme o aumento da idade, cresce o número de idosos com algum grau de incapacidade, afirmação essa justificada pelos autores por meio dos resultados esboçados em seus estudos, visto que idosos com idade acima de 75 anos apresentaram cerca de oito vezes mais probabilidade de ter sua mobilidade comprometida no TUG, quando comparados aos indivíduos da terceira idade com menos de 75 anos. Ademais, cerca de 50\% daqueles com faixa etária maior de 85 anos apresentaram limitações em suas AVD's, enquanto que outros $50 \%$ foram classificados como sendo dependentes. Em contrapartida, para Ferratin et al.29, o aumento da idade por si só não constitui um fator limitante para a realização das AVD's e da mobilidade funcional, destacando também que, quanto maior for o grau de dependência do indivíduo, piores serão os impactos sobre o seu bem-estar e qualidade de vida. À vista disso, pode-se depreender que risco de quedas, funcionalidade e equilíbrio na população idosa em geral são considerados fatores diretamente relacionados entre $\mathrm{si}^{28}$.

Quando correlacionados o TAF, o TUG e a idade, observou-se que aquele indivíduo que obteve melhores índices no TAF também obteve melhores índices no TUG, apresentando baixo risco de quedas, e era mais jovem. Correlação essa justificada também em um estudo de Almeida et al. ${ }^{30}$, em que concluíram que o avanço da idade está associado a valores crescentes no TUG e valores decrescentes do TAF, ou seja, quanto maior a idade, maior o risco de quedas. Segundo Álvares et al. ${ }^{31}$, tal explicação é afirmada devido, provavelmente, à diminuição da qualidade e quantidade de informações necessárias para um eficiente controle postural.

O presente estudo teve como principal limitação o fato de as análises terem sido coletadas diante de idosos participantes de apenas um dos centros de convivência da região, tornando-se assim uma discussão na qual não se pode generalizar os resultados obtidos para todos os idosos que participam desses projetos sociais. Entretanto, a partir da vivência desse grupo de estudo, participar de atividades oferecidas nesses centros como ginástica, dinâmica de grupos, dança, jogos de 
raciocínio lógico, proporciona uma sensação de prazer e é capaz de reestabelecer vínculos sociais, além de contribuir diretamente para o processo de senescência saudável.

\section{CONCLUSÃO}

A pesquisa realizada mostrou relevância durante a correlação das variáveis aplicadas aos participantes, em relação à Escala de Lawton, TAF, TUG e ao fator idade. Â vista disso, destaca-se que o avanço da idade, a capacidade funcional, o equilíbrio, a mobilidade e o risco de quedas estão diretamente associados uns com os outros.

Levando em consideração a população estudada, bem como seu estilo de vida adotado, observou-se que os indivíduos que apresentaram idade inferior eram mais independentes nas suas AVD's, além de apresentarem uma mobilidade funcional melhor e menor risco de quedas.

Estima-se a partir deste estudo e das demais referências utilizadas para construção do mesmo que, idosos inseridos em grupos de convivência apresentam maiores chances de ter resultados satisfatórios em testes funcionais. Afirmação pertinente devido ao fato de se tratar de indivíduos mais ativos fisicamente e socialmente, o que proporciona, consequentemente, melhora na qualidade de vida, diminuição do índice de dependência de cuidados, além da busca pelo envelhecimento saudável.

\section{REFERÊNCIAS}

1. Camarano AA. (Org.). Muito além dos 60: os novos idosos brasileiros. Rio de Janeiro: Ipea; 2004.

2. Organização Pan-Americana de Saúde. Envelhecimento ativo: uma política de saúde. Brasília (DF); 2005.

3. Wichmann FMA, Couto AN, Areosa SVC, et al. Grupos de convivência como suporte ao idoso na melhoria da saúde. Rev Bras Geriat Gerontol. 2013;16(4):821-32.

4. Andrade TP, Mendonça BPCK, Lima DC, et al. Projeto Conviver: Estímulo à Convivência entre Idosos do Catete, Ouro Preto, MG. Rev Bras Educ Méd. 2012;36(1):81-5.

5. Rizzolli D, Surdi AC. Percepção dos idosos sobre grupos de terceira idade. Rev. Bras. Geriatr Gerontol. 2010;13(2): 225-33.

6. Del Duca GF, Silva MC, Hallal PC. Incapacidade funcional para atividades básicas e instrumentais da vida diária em idosos. Rev Saúde Públ. 2009;43(5):796-805.

7. Barbosa BR, Almeida JM, Barbosa MR, et al. Avaliação da capacidade funcional dos idosos e fatores associados à incapacidade. Ciênc Saúde Colet. 2014;19(8)3317-25.

8. Gonçalves CMM. A qualidade de vida em idosos institucionalizados. Tese de Doutorado; 2015.

9. Gonçalves R, Gurjão ALD, Gobbi S. Efeitos de oito semanas do treinamento de força na flexibilidade de idosos. Rev Bras Cineantropom Hum. 2007; 9(2):145-53.
10. Ribeiro AP, Souza ER, Atie S, et al. A influência das quedas na qualidade de vida de idosos. Ciênc Saúde Colet. 2008; 13(4):1265-73.

11 Celich KLS, Souza SMS, Zenevics L, et al. Fatores que predispõem às quedas em idosos. RBCEH. 2010;7(3): 419-26.

12. Lawton, M P, Brody, MH. Assessment of older people: Selfmaintaining and instrumental activities of daily living. The Gerontologist. 1969;9(3):179-86.

13. Brasil. Ministério da Saúde. Secretaria de Atenção à Saúde. Departamento de Atenção Básica. Envelhecimento e saúde da pessoa idosa. Brasília (DF); 2006.

14. Karuka AH, Silva JAMG, Navega MT. Análise da concordância entre instrumentos de avaliação do equilíbrio corporal em idosos. Rev Bras Fisioter. 2011;15(6):460-6.

15. Gomes GS. Tradução, adaptação transcultural e exame das propriedades de medida da escala "Performance-Orientes Mobility Assessment"(POMA) para uma amostra de idosos brasileiros institucionalizados [Dissertação]. Campinas (SP): Universidade Estadual de Campinas; 2003.

16. Podsiadlo D, Richardon S. The timed "Up \& Go": a test of basic functional mobility for frail elderly pearsons. J Am Geriatr Soc. 1991;39(2):142-8.

17. Bischoff HA, Stähelin HB, Monsch AU, et al. Identifying a cut-off point for normal mobility: A comparison of the timed 'up and go' test in community-dwelling and institutionalised elderly women. Age Ageing. 2003;32(3):315-20.

18. Brasil. Ministério da Saúde (MS). Secretaria de Atenção à Saúde. Departamento de Ações Programáticas e Estratégicas. Atenção à saúde da pessoa idosa e envelhecimento. Brasília (DF); 2010.

19. Miranda LCV, Soares SM, Silva PAB. Qualidade de vida e fatores associados em idosos de um Centro de Referência à Pessoa Idosa. Ciênc Saúde Colet. 2016;21(11):3533-44.

20. Costa LSV, Sousa NM, Alves AG, et al. Análise comparativa da qualidade de vida, equilíbrio e força muscular em idosos praticantes de exercício físico e sedentários. Rev Facul Montes Belos. 2015;8(3):61-179.

21. Celich KLS, Galon C. Dor crônica em idosos e sua influência nas atividades da vida diária e convivência social. Rev Bras Geriat Gerontol. 2009;12(3):345-59.

22. Gomercindo MCH, Garcez EMS. Avaliação da capacidade funcional de idosos de uma comunidade do município de Porto União em Santa Catarina. Rev. Saúde Públ. Santa Catarina 2012; 5(2):30 - 45.

23. Barbosa MH, Bolina AF, Tavares JL, et al. Sociodemographic and health factors associated with chronic pain in institutionalized elderly. Rev Latinoam Enfermagem. 2014; $22(6): 1009-16$

24. Campos MPS, Vianna LG, Campos AR. Os testes de equilíbrio Alcance Funcional e "Timed Up and Go" e o risco de quedas em idosos. Revista Kairós Gerontologia. 2013; 16(4):125-38.

25. Maciel ACC, Araújo LM. Fatores associados como alterações na velocidade de marcha e força de preensão manual de em idosos institucionalizados. Rev Bras Geriat Gerontol. 2010; 13(2):179-89.

26. Lustosa LP, Marra TA, Pessanha FPAS, et al. Fragilidade e funcionalidade entre idosos frequentadores de grupos de convivência em Belo Horizonte, MG. Rev Bras Geriat Gerontol. 2013; 16(2):347-54.

27. Guerra HS, Bernardes DCF, Santana JA, et al. Avaliação do risco de quedas em idosos da comunidade. Rev Saúde Com. 2017; 13(2):879-86. 
28. Souza CC, Valmorbida LA, Oliveira JP, et al. Mobilidade funcional em idosos institucionalizados e não institucionalizados. Rev Bras Geriatr Gerontol. 2013;16(2):285-93.

29. Ferratin AC, Borges CF, Morelli JGS, et al. A execução de AVDS e mobilidade funcional em idosos institucionalizados e não-institucionalizados. Fisioter Mov. 2007;20(3): $115-21$.
30. Almeida ST, Soldera CLC, Carli GA, et al. Análise de fatores extrínsecos e intrínsecos que predispõem a quedas em idosos. Rev Assoc Med Bras. 2012; 58(4):427-33.

31. Álvares LM, Lima RC, Silva RA. Ocorrência de quedas em idosos residentes em instituições de longa permanência em Pelotas, Rio Grande do Sul, Brasil. Cad Saúde Pública. 2010; 26(1):31-40.

\section{AUTORES}

GABRIELA OLIBONIFIORIO <fiorio.gabriela@gmail.com>

Graduada em Fisioterapia, Centro Universitário da Serra Gaúcha (FSG), Caxias do Sul, RS, Brasil.

(D) orcid.org/0000-0001-6532-0310

GISELE OLTRAMARI MENEGHINI < gisele.oltramari@fsg.br>

Graduada em Fisioterapia, ULBRA. Mestre em Gerontologia Biomédica. Docente do curso de Fisioterapia do Centro Universitário da Serra Gaúcha (FSG),

Caxias do Sul, RS, Brasil. 